\title{
Azlocilina y amikacina: ¿una alternativa terapéutica para la septicemia por estafilococos resistentes?
}

\author{
María Espino Hernández, ${ }^{1}$ María Julia Couto Ramos ${ }^{2}$ \\ y Niurka Fiol Ferrer ${ }^{3}$
}

Forma de citar Espino Hernández M, Couto Ramos MJ, Fiol Ferrer N. Azlocilina y amikacina: ¿una alternativa terapéutica para la septicemia por estafilococos resistentes? Rev Panam Salud Publica. 2004;16(5):315-9.

RESUMEN Objetivo. Evaluar la eficacia de la combinación de azlocilina y amikacina en un grupo de recién nacidos con septicemia causada por estafilococos multirresistentes, internados en la unidad de cuidados intensivos neonatales (UCIN) del Hospital Ginecobstétrico "América Arias", de La Habana, Cuba, en el período comprendido entre 1998 y 2000.

Métodos. Se realizó un análisis retrospectivo de los resultados clínicos y de laboratorio obtenidos en 15 pacientes con septicemia causada por estafilococos multirresistentes que recibieron tratamiento con azlocilina más amikacina según la estrategia trazada en el hospital para el uso de antimicrobianos. Se evaluó el patrón de resistencia de las cepas frente a 10 antibióticos en uso mediante el método de microdilución en caldo. En ocho de los pacientes tratados se vigilaron los efectos del tratamiento mediante pruebas de sinergia in vitro, por el método del "tablero de damas" en bandejas de microtitulación.

Resultados. Se aislaron 12 estafilococos coagulasa-negativos y tres Staphylococcus aureus, en los que se identificaron cinco patrones distintos de resistencia con base en la susceptibilidad mostrada a la oxacilina, a tres aminoglucósidos y a la vancomicina. En seis de las pruebas de sinergia realizadas se obtuvo un notable efecto sinérgico, con disminución promedio de tres diluciones en la CMI en el caso de los dos antibióticos utilizados en el tratamiento. Ningún resultado fue antagónico y la eficacia clínica general de la combinación alcanzó 91,7 por ciento. Conclusiones. El ensayo demostró la eficacia de la combinación de antibióticos empleada, si bien se reconoce la necesidad de ampliar las investigaciones antes de poder emitir resultados totalmente concluyentes.

Palabras clave Staphylococcus, septicemia, neonato, resistencia a múltiples drogas, sinergismo de drogas, combinación de medicamentos, antibióticos combinados, azlocilina, amicacina (amikacina).

1 Departamento de Agentes Biológicos, Escuela Latinoamericana de Medicina, Ciudad de La Habana. Toda correspondencia deberá dirigirse a: María Espino Hernández, Departamento de Agentes Biológicos, Escuela Latinoamericana de Medicina. Km 31/2 Carretera Panamericana, Santa Fe, Playa, Ciudad de la Habana, Cuba. Teléfono: (537)
209 7477, ext. 568. Fax: (537) 33 1025. Correo electrónico: mespino@elacm.sld.cu

2 Departamento de Neonatología, Hospital Ginecobstétrico "América Arias".

3 Departamento de Microbiología, Hospital Ginecobstétrico "América Arias".
Las bacterias grampositivas son los microorganismos de mayor prevalencia en las unidades de cuidados intensivos neonatales (UCIN). En particular, los estafilococos coagulasanegativos $(\mathrm{ECoN})$ se han señalado en 
los últimos años como causa de infección tardía en más de $50 \%$ de los recién nacidos internados en UCIN —en su mayoría sometidos a maniobras invasoras-, lo que implica un incremento considerable del tiempo y los costos de hospitalización (1).

La multirresistencia a antibióticos es común en estos microorganismos $\mathrm{y}$ se informa en todas partes del mundo. La susceptibilidad se limita prácticamente a la vancomicina y algunos otros fármacos de reciente introducción (2).

La vancomicina es un antibiótico con gran acción bactericida contra estafilococos y otros microorganismos grampositivos. No obstante, a pesar de haberse descubierto en 1955 e introducido en la práctica clínica mucho antes que las penicilinas resistentes a las penicilinasas, el uso de este fármaco se vio limitado por su reconocido alto grado de toxicidad (3). Sin embargo, ante la situación alarmante que significa la resistencia microbiana que se observa a escala mundial, este antibiótico volvió a captar interés y hoy se recomienda como primera elección terapéutica en casos de infecciones por estafilococos resistentes y de otras similares (1). A causa del uso extenso y el abuso que se ha hecho del fármaco en los últimos años, en el decenio de 1980 comenzaron a aparecer las primeras cepas resistentes al compuesto. Las especies de estafilococos coagulasanegativos resistentes a la meticilina y a la vancomicina (ECoNMR-VR), junto a las de Enterococcus resistentes a la vancomicina (EVR), se convirtieron en poco tiempo en una verdadera contingencia en el ámbito hospitalario a finales de ese mismo decenio. Este hecho constituyó motivo de alerta, por el peligro que representaría la extensión de esta característica a agentes patógenos potenciales, como Staphylococcus aureus $(2,4)$. Los primeros informes de una disminución de la susceptibilidad a la vancomicina en cepas de $S$. aureus resistente (SARM) a la meticilina se dieron a finales del decenio de 1990, y en 2002 se notificaron los dos primeros aislamientos resistentes, obtenidos de las secreciones de heridas quirúrgicas de pacientes hospitalizados (5-7).

Ante el impacto que el fenómeno de la resistencia ha tenido en los indica- dores de salud y en los costos, resulta evidente la necesidad de modificar las prácticas de tratamiento, en el sentido de hacer un uso racional de los antimicrobianos y de buscar nuevas estrategias que ayuden a preservar la eficacia de los antibióticos ya existentes (8).

En el presente trabajo se analizan los resultados que se obtuvieron al administrar la combinación de los antibióticos azlocilina y amikacina en un grupo de recién nacidos que sufrieron septicemia por estafilococos multirresistentes. Se detallan el patrón de resistencia de las cepas procedentes de estos pacientes, los datos de laboratorio obtenidos acerca de la interacción sinérgica entre los fármacos utilizados contra un grupo de los microorganismos aislados, y se comparan estos datos con los resultados clínicos logrados.

\section{MÉTODOS}

Se realizó un análisis retrospectivo de los resultados clínicos y de laboratorio obtenidos en 15 pacientes con septicemia por estafilococos multirresistentes, tratados con una combinación de azlocilina y amikacina de acuerdo con la estrategia para el uso de antimicrobianos establecida en la UCIN de este centro hospitalario, en el período comprendido entre 1998 y 2000.

Se realizó una caracterización de los pacientes a partir del cuadro clínico (signos y síntomas más frecuentes), el tipo de sepsis y el peso al nacimiento. También se determinó la dosis de cada uno de los fármacos administrados, expresada en $\mathrm{mg} / \mathrm{kg}$ por día o por dosis.

Las cepas fueron aisladas de cultivos duplicados de muestras de sangre obtenidas de venas periféricas antes de la aplicación del tratamiento antimicrobiano, respetando las condiciones de asepsia requeridas (empleo de guantes y desinfección de la zona por puncionar).

Se evaluó el patrón de resistencia de los aislamientos con respecto a 10 antibióticos en uso en el hospital, a saber: penicilina G, azlocilina, cefazolina, cefotaxima, ceftriaxona, kanamicina, gentamicina, amikacina, vancomicina y oxacilina (utilizada como indicador de la resistencia a meticilina).
El método empleado fue el de microdilución en caldo, según las normativa establecida por el National Committee for Clinical Laboratory Standards (NCCLS) $(9,10)$. Se utilizaron soluciones estándares de laboratorio de potencia conocida para preparar las soluciones patrón de los antibióticos. Se determinaron las concentraciones mínimas inhibidoras (CMI) dentro de un intervalo de 0,125 a $128 \mu \mathrm{g} / \mathrm{mL}$. El control de calidad se realizó con las cepas de referencia S. aureus ATCC 29213, Escherichia coli ATCC 25922 y Pseudomonas aeruginosa ATCC 27853.

Se analizaron los resultados obtenidos en ocho de los pacientes, cuyos tratamientos fueron monitorizados mediante pruebas de sinergia in vitro. Se empleó el método del "tablero de damas" (checkerboard), el cual se llevó a cabo en bandejas de microtitulación (10). El panel de diluciones para la combinación de antibióticos ensayada se preparó a partir de una concentración de $128 \mu \mathrm{g} / \mathrm{mL}$. Se evaluó la posibilidad de sinergia usando combinaciones de los dos fármacos a diferentes concentraciones. Los resultados se evaluaron mediante el cálculo de los índices de concentración fraccionaria inhibidora (CFI), como sigue:

$\mathrm{CFI}=\mathrm{CFI}_{\mathrm{A}}+\mathrm{CFI}_{\mathrm{B}}$,

donde:

$\mathrm{CFI}_{\mathrm{A}}=\frac{\mathrm{CMI}_{\mathrm{A}+\mathrm{B}}}{\mathrm{CMI}_{\mathrm{A}} \text { solo }}$

$\mathrm{CFI}_{\mathrm{B}}=\frac{\mathrm{CMI}_{\mathrm{A}+\mathrm{B}}}{\mathrm{CMI}_{\mathrm{B}} \text { solo }}$

$\mathrm{CMI}_{\mathrm{A}+\mathrm{B}}=\mathrm{CMI}$ de la combinación de $\mathrm{A}$ con $\mathrm{B}$.

Se consideró que había sinergia si el CFI era $\leq 0,500$; sinergia baja o parcial si $0,500<\mathrm{CFI}<1$; indiferencia o adición cuando $1 \leq \mathrm{CFI}<2$, y antagonismo si CFI $\geq 2$.

Los criterios para determinar la eficacia del tratamiento fueron los siguientes:

- Tratamiento eficaz: cuando al concluir el tratamiento por el tiempo establecido desaparecieron totalmente los signos y síntomas de infección en el paciente y pudo com- 
probarse, por los estudios microbiológicos correspondientes (hemocultivo), la ausencia total de proliferación microbiana.

- Tratamiento ineficaz: cuando el tratamiento tuvo que alterarse mediante la sustitución de por lo menos uno de los fármacos de la combinación o cuando a pesar de haber concluido el esquema de tratamiento pudo comprobarse, por los estudios microbiológicos, la presencia del mismo agente causal con un patrón de susceptibilidad igual o similar, acompañado o no este resultado de la persistencia de síntomas y signos.

\section{RESULTADOS}

El cuadro clínico de los pacientes se caracterizaba por dificultad respiratoria, más fiebre o distermia en todos los casos, y por uno o más de los signos y síntomas siguientes: ictericia, cianosis, livedo reticularis, vómito y producción aumentada del residuo gástrico. Una proporción de $66,6 \%$ de los recién nacidos (10 del total) tuvo un peso al nacer inferior a $2500 \mathrm{~g}$ (promedio: 2 016,4 g). Los 15 pacientes tenían colocada una vía parenteral y cuatro estaban acoplados al respirador. Se diagnosticaron 10 septicemias generalizadas (siete adquiridas y tres connatales) y cinco bronconeumonías (tres adquiridas y dos connatales).

Se aislaron tres $S$. aureus y $12 \mathrm{ECoN}$ productores de biopelícula (slime), todos los cuales resultaron resistentes a la oxacilina, con valores de CMI entre 16 y $3128 \mu \mathrm{g} / \mathrm{mL}$. Cuatro de estas cepas fueron resistentes también a la vancomicina (CMI $3128 \mu \mathrm{g} / \mathrm{mL}$ ). Se identificaron cinco patrones distintos de resistencia, clasificados con base en los resultados correspondientes a oxacilina, vancomicina y los aminoglucósidos probados. A estos patrones se les asignaron ordinales romanos del I al V. En el cuadro 1 se señala la resistencia porcentual encontrada con cada uno de los antibióticos, así como el intervalo de valores de la CMI, acompañado del valor de $\mathrm{CMI}_{50}$ (concentración mínima que inhibió a $50 \%$ de los aislamientos). En el cuadro 2 se deta- llan las características de los patrones de resistencia identificados y el total de aislamientos en cada uno de los casos.

En los resultados de la monitorización del tratamiento realizada en el transcurso de las pruebas de sinergia se observó sinergia en seis de los ocho ensayos efectuados, lo que equivale a $75 \%$. En lo referente a estos casos, el intervalo de la CFI osciló entre 0,015 y 0,500 y se constató una disminución promedio de tres diluciones en los valores de CMI con los dos antibióticos en la combinación. En un ensayo se obtuvo sinergia baja o parcial $(\mathrm{CFI}=0,750)$ e indiferencia en el resto $(\mathrm{CFI}=1,060)$. Todos fueron congruentes con un resultado terapéutico clínico satisfactorio (cuadro 3).

Las dosis de los antibióticos administrados oscilaron dentro de un intervalo de 125 a $300 \mathrm{mg} / \mathrm{kg}$ de azlocilina, distribuidos en tres o cuatro dosis por día, y de 5 a $7 \mathrm{mg} / \mathrm{kg}$ de amikacina. En 14 pacientes el tratamiento aplicado mostró eficacia y en uno no fue eficaz. La eficacia general de la combinación fue de $91,7 \%$.

\section{DISCUSIÓN}

La circulación de SARM y estafilococos coagulasa-negativos multirresistentes (ECoNMR) en las unidades hospitalarias de la capital cubana se demostró por primera vez en dos estudios, uno multicéntrico y otro realizado en el Hospital Ginecobstétrico "América Arias", entre los años 1994 y 1995. En este último ya se declaraba entonces a estos microorganismos como los principales agentes causales de septicemia en la $\operatorname{UCIN~}(11,12)$.

El antibiótico vancomicina se introdujo en el país al principio del decenio de 1990 y desde el primer momento

CUADRO 1. Resistencia en las cepas de estafilococos aisladas de los recién nacidos sépticos. Total de aislamientos: 15

\begin{tabular}{|c|c|c|c|c|}
\hline Antibiótico & $\begin{array}{c}\text { Cepas } \\
\text { resistentes }\end{array}$ & $\begin{array}{c}\text { Resistencia } \\
(\%)\end{array}$ & $\begin{array}{l}\text { Intervalo CMI } \\
(\mu \mathrm{g} / \mathrm{mL})\end{array}$ & $\begin{array}{c}\mathrm{CMI}_{50} \\
(\mu \mathrm{g} / \mathrm{mL})\end{array}$ \\
\hline Penicilina G & 15 & 100 & {$[4 \mathrm{a} \geq 128]$} & $\geq 128$ \\
\hline Oxacilina & 15 & 100 & {$[8 \mathrm{a} \geq 128]$} & $\geq 128$ \\
\hline Azlocilina & 15 & 100 & {$[8 \mathrm{a} \geq 128]$} & $\geq 128$ \\
\hline Cefazolina & 15 & 100 & {$[32 \mathrm{a} \geq 128]$} & $\geq 128$ \\
\hline Cefotaxima & 15 & 100 & {$[32 \mathrm{a} \geq 128]$} & $\geq 128$ \\
\hline Ceftriaxona & 15 & 100 & {$[64 \mathrm{a} \geq 128]$} & $\geq 128$ \\
\hline Kanamicina & 15 & 100 & {$[32 \mathrm{a} \geq 128]$} & $\geq 128$ \\
\hline Gentamicina & 14 & 93,3 & {$[1 \mathrm{a} \geq 128]$} & 32 \\
\hline Amikacina & 8 & 53,3 & {$[1 \mathrm{a} \geq 128]$} & 32 \\
\hline Vancomicina & 4 & 26,6 & {$[1 \mathrm{a} \geq 128]$} & 2 \\
\hline
\end{tabular}

CMI: concentración mínima inhibidora; $\mathrm{CMI}_{50}$ : concentración mínima que inhibe a $50 \%$ de los aislamientos.

\section{CUADRO 2. Descripción de los patrones de resistencia encontrados}

\begin{tabular}{clccc}
\hline No. & \multicolumn{1}{c}{ Patrón de resistencia } & ECoN & SA & Total \\
\hline I & $\begin{array}{l}\text { Resistente a oxacilina y kanamicina. Susceptible } \\
\text { a gentamicina, amikacina y vancomicina }\end{array}$ & 0 & 1 & 1 \\
V & $\begin{array}{l}\text { Resistente a oxacilina, kanamicina y gentamicina. } \\
\text { Susceptible a amikacina y vancomicina }\end{array}$ & 3 & 1 & 4 \\
IV & $\begin{array}{l}\text { Resistente a oxacilina, kanamicina, gentamicina } \\
\text { y amikacina. Susceptible a vancomicina }\end{array}$ & 5 & 1 & 6 \\
IV & $\begin{array}{l}\text { Resistente a oxacilina, kanamicina, gentamicina } \\
\text { y vancomicina. Susceptible a amikacina }\end{array}$ & 2 & 0 & 2 \\
V & Resistente a todos los antibióticos probados & 2 & 0 & 2 \\
\hline ECoN: estafilococos
\end{tabular}


CUADRO 3. Resultados in vitro de la interacción de los fármacos frente a los aislamientos estudiados en ocho ensayos

\begin{tabular}{lcrrcccc}
\hline Aislamiento & Patrón & $\mathrm{CMI} \mathrm{A}_{1}$ & $\mathrm{CMI} \mathrm{A}_{2}$ & $\begin{array}{c}\mathrm{CMI} \mathrm{A}_{1} / \\
\mathrm{CMI} \mathrm{A}_{2}\end{array}$ & $\begin{array}{c}\text { Índice } \\
\mathrm{CFI}\end{array}$ & $\begin{array}{c}\text { Resultado } \\
\text { eficaz }\end{array}$ \\
\hline ECoNMR & III & $\geq 128$ & $\geq 128$ & $2 / 0,06$ & 0,015 & $\mathrm{~S}$ & Sí \\
ECoNMR & III & 16 & 32 & $2 / 1$ & 0,156 & $\mathrm{~S}$ & Sí \\
ECoNMR & IV & 128 & 1 & $32 / 0,25$ & 0,500 & $\mathrm{~S}$ & Sí \\
ECoNMR & III & 8 & 64 & $2 / 4$ & 0,312 & $\mathrm{~S}$ & Sí \\
ECoNMR & IV & 64 & 1 & $64 / 0,06$ & 1,060 & $\mathrm{I}$ & Sí \\
ECoNMR & V & $\geq 128$ & 32 & $32 / 16$ & 0,750 & $\mathrm{SB}$ & Sí \\
ECoNMR & II & 128 & 2 & $32 / 0,50$ & 0,500 & $\mathrm{~S}$ & Sí \\
SARM & III & 64 & 32 & $16 / 8$ & 0,500 & $\mathrm{~S}$ & Sí \\
\hline
\end{tabular}

$\mathrm{A}_{1}$ : antibiótico 1 (azlocilina); $\mathrm{A}_{2}$ : antibiótico 2 (amikacina); CFI: concentración fraccionaria inhibidora; CMI: concentración mínima inhibidora; ECoNMR: estafilococos coagulasa-negativos resistentes a meticilina; SARM: Staphylococcus aureus resistente a meticilina; Tx: tratamiento.

pasó a formar parte del grupo de los antibióticos regulados a instancia nacional para uso exclusivo en pacientes graves con sepsis por estafilococos resistentes. No obstante, en menos de un decenio pudo comprobarse, en este centro hospitalario, la aparición de resistencia al compuesto en cepas de ECoN, un proceso que se piensa tuvo como punto de partida una cepa de Enterococcus faecium procedente de un neonato séptico internado en la UCIN, durante el mes de septiembre de 1997. Al constatarse después en dicha cepa la presencia del gen Van A, se consideró la posibilidad de transferencia horizontal de esta característica a las cepas de ECoN, lo cual comenzó a ocurrir al poco tiempo $(13,14)$.

La detección, en el mes de febrero de 1998, de un recién nacido con un cuadro de bronconeumonía adquirida por Staphylococcus haemolyticus multirresistente (resistencia comprobada a 30 antibióticos), quien curó con un tratamiento basado en la combinación de azlocilina y amikacina, constituyó el punto de partida de estos ensayos y de la aplicación ulterior de igual régimen terapéutico en pacientes con cuadros clínicos similares (15).

En general, todas la cepas resultaron muy resistentes a los antibióticos probados, para los cuales la CMI en 50\% de los casos fue $\geq 128 \mu \mathrm{g} / \mathrm{mL}$, aunque se encontró también una respuesta variable en cuanto a la resistencia a aminoglucósidos, lo cual se explica a la luz de los muchos mecanismos plan- teados en torno a la resistencia a estos compuestos (16).

Los ensayos in vitro con combinaciones de los diferentes fármacos de uso actual han atraído la atención de diferentes grupos de investigadores de todo el mundo que buscan posibles alternativas terapéuticas para tratar infecciones graves causadas por bacterias muy resistentes. Si bien abundan los informes de la eficacia in vitro de diferentes combinaciones de antibióticos, son sumamente escasos los estudios que demuestren una correspondencia de esos resultados con la eficacia in vivo de ese régimen terapéutico.

Las combinaciones probadas con mayor frecuencia se sustentan en el conocido efecto sinérgico que supone la interacción entre un antibiótico $B$ lactámico y un aminoglucósido. En lo que se refiere específicamente al tratamiento de infecciones por SARM y ECoNMR, los informes han sido variados $(17,18)$. No obstante, con excepción de los datos anteriores, notificados ya antes por los autores de este trabajo, no se ha encontrado ninguna experiencia anterior, clínica o de laboratorio, con el uso de la combinación de azlocilina y amikacina contra estos microorganismos en particular $(14,15)$.

La azlocilina es un derivado de la ampicilina, con un radical acilureídico en la cadena lateral del anillo $\beta$ lactámico que dota al antibiótico de una fuerte actividad bactericida de amplio espectro. A este fármaco se le reconoce gran actividad contra especies de Pseudomonas y de Haemophilus y contra otros bacilos gramnegativos, aunque se considera ineficaz contra estafilococos productores de ß-lactamasa (19). Al igual que el resto de las penicilinas, tiene muy baja toxicidad, a no ser la relativa que se observa en pacientes que sufren alergia a estos compuestos o a algunos de sus metabolitos. De hecho, esta característica supone una gran ventaja en el recién nacido, porque permite hacer uso de dosis altas cuando es necesario. No obstante, en el grupo de pacientes tratados, la dosis máxima suministrada fue de $300 \mathrm{mg} / \mathrm{kg}$ al día, en combinación con una dosis adecuada de amikacina (5 a $7 \mathrm{mg} / \mathrm{kg}$ ), acorde al riesgo conocido de toxicidad de los aminoglucósidos en general.

Llama la atención que se haya obtenido sinergia baja o parcial frente a una de las cepas con patrón $\mathrm{V}$ (resistente a todos los antibióticos), contra la cual resultó también satisfactorio el tratamiento del paciente, mientras que, por otro lado, el único resultado de ineficacia terapéutica se observó en otro paciente afectado por una cepa similar. Ambas cepas se diferenciaron únicamente en la resistencia contra amikacina, que en el último fue $\geq 128 \mu \mathrm{g} / \mathrm{mL}$. De hecho, solo dos aislamientos presentaron este valor, ya que en las otras cepas resistentes al compuesto la CMI no pasó de $64 \mu \mathrm{g} / \mathrm{mL}$. Este dato podría constituir una demostración de que la eficacia de la combinación depende de valores de resistencia bajos o de susceptibilidad intermedia en lo que atañe al antibiótico aminoglucósido. No obstante, los resultados que aquí se presentan permiten concluir que la combinación de azlocilina y amikacina, aplicada en dosis similares a las que suelen emplearse en el recién nacido, puede constituir un tratamiento alternativo al uso de vancomicina en la septicemia por estafilococos resistentes. No obstante, para poder emitir resultados totalmente concluyentes es necesario profundizar en los estudios in vitro con cepas que posean niveles de resistencia contra la amikacina superiores al indicado por la CMI de $128 \mu \mathrm{g} / \mathrm{mL}$ evaluada aquí. 


\section{REFERENCIAS}

1. Isaac D. A ten-year multicentre study of coagulase-negative staphylococcal infections in Australasian neonatal units. Arch Dis Fetal Neontal. 2003;88:F89-F93.

2. Kollef $\mathrm{MH}$. Antibiotic resistance among Gram-positive bacteria in the hospital setting: what can we do about it? [Sitio de Internet] Medscape, Inc. Hallado en: http://www. medscape.com/viewprogram/623. Acceso el 10 de diciembre de 2002.

3. Colectivo de autores. Update on antibiotics II. Med Clin North Am. 1988;72(3):555-743.

4. Schwalbe RS, Stapleton JT, Gilligan PH. Emergence of vancomycin resistance in coagulase negative Staphylococci. N Engl J Med. 1987; 316:927-31.

5. Public Health Dispatch: Vancomycin-resistant S. aureus-Pennsylvania, 2002. MMWR Morb Mortal Wkly Rep. 2002;51:902.

6. Centers for Disease Control and Prevention. S. aureus resistant to vancomycin-United States, 2002. MMWR Morb Mortal Wkly Rep. 2002;51:565-7.

7. Hersberger E, Hayden M, Snydman DR, et al. Multicenter surveillance for staphylococcal isolates with diminished susceptibility to vancomycin. Presented at the 40th IDSA annual meeting; October 24-27, 2002. Chicago, Illinois. Poster 93.
8. Smith RD, Coast J. Antimicrobial resistance: a global response. Bull World Health Organ. 2002;80:126-32.

9. National Committee for Clinical Laboratory Standards. Performance Standards for Antimicrobial Susceptibility Testing-Twelfth Informational Supplement, M100-S12. Wayne (PA): NNCLS; 2002;22.

10. National Committee for Clinical Laboratory Standards. Method for dilution antimicrobial susceptibility test for bacteria that grow aerobically. Wayne (PA): NCCLS; 1993. (Document M7-A3, Supplement 1).

11. Niebla A, Espino M, González I, Otero I, Lemus M, González L. Oxacillin resistance in staphylococcus clinical isolates from Havana City Hospital, Cuba. Rev CENIC. 2001;32: 33-6.

12. Espino M, Couto MJ, Lee M, Páez N, Meriño E. Efecto sinérgico de penicilina G y kanamicina en septicemia neonatal por estafilococos. Rev Cubana Pediatr. 1995;67:155-64.

13. González L, González I, Espino M, Garmendía L, Nodal L, Lemus M. Identificación y susceptibilidad a antibióticos en cepas de Enterococcus spp. en el Hospital Ginecobstétrico América Arias. Rev Latinoam Microbiol. OctDic 2002;44:572S.

14. Espino M, Couto MJ, Fiol N, Rojas N. Resistencia a antimicrobianos y evaluación del tra- tamiento combinado en la septicemia neonatal. Pan Am J Public Health. 2003;13:214-21.

15. Espino M, Fiol N, Lee M, Couto MJ. Tratamiento con azlocilina y amikacina en septicemia neonatal por $S$. haemolyticus multirresistente. Rev Cubana Pediatr. 2000;72:60-4.

16. Moreira BM, Daum RS. Antimicrobial Resistance in Staphylococcus. Pediatr Clin North Am. 1995;42:619-39.

17. Oka S, Gato M, Kaji Y, Kimura S, Matsuda K, Asahi Y. Synergic activity of imipenem/ cilastin combined with cefotian against methicillin resistant $S$. aureus. J Antimicrob Agents Chemother. 1993;31:533-41.

18. Archer KN, Climo MW. Combination of lisostaphin with $\beta$-lactams are synergistic again oxacillin-resistant Staphylococcus epidermidis. Antimicrob Agents Chemother. 2002; 46:2017-20

19. Ruiz Palacios G, Sifuentes JO, Ponce de León SR, Calva JJM. Antibioticoterapia bacteriana (fascículo), 2ª ed. México, D.F.: Obsidiana; 1986.

Manuscrito recibido el 3 de noviembre de 2003. Aceptado para publicación, tras revisión, el 8 de septiembre de 2004.

ABSTRACT Objective. To assess the effectiveness of combined therapy with azlocillin and amikacin in a group of neonates with sepsis caused by multiresistant staphylococci who were hospitalized in the neonatal intensive care unit of Hospital Ginecobstétrico "América Arias" in Havana, Cuba, from 1998 to 2000.

\section{Azlocillin plus amikacin: an alternative therapy for sepsis caused by resistant staphylococci?}

Methods. A retrospective study was carried out of the clinical and laboratory results obtained in 15 patients with sepsis caused by multiresistant staphylococci who received combined therapy with azlocillin and amikacin, according to hospital guidelines on the use of antibiotics. We used a broth microdilution method to study the patterns of resistance shown by isolated strains to 10 of the antibiotics in use. In vitro synergy tests, specifically the checkerboard technique with microtitration plates, were used to observe the effects of treatment in 8 patients.

Results. Twelve coagulase-negative staphylococci and three Staphylococcus aureus isolates showed five different patterns of resistance on the basis of their sensitivity to oxacillin, three aminoglycosides, and vancomycin. Six of the synergy tests showed a considerable synergistic effect, with an average three-fold reduction in the minimum inhibitory concentrations (MIC) of the two antibiotics used to treat the patients. No antagonistic effects were noted, and the combined antibiotics showed an overall clinical effectiveness of $91.7 \%$.

Conclusions. The test showed that the therapeutic combination used was effective, but further studies are needed before conclusive results are obtained. 